\title{
ANOMALOUS REDSHIFTS IN GROUPS OF GALAXIES
}

\section{J. HEIDMANN}

Observatoire de Paris, F-92190, Meudon

Nous passons en revue les données d'observation sur les décalages spectraux anormaux dans les groupes de galaxies, incluant les paires, mais laissant de côté les aspects traités par d'autres : associations galaxie-quasar, amas de galaxies et théories.

La première partie traite des études individuelles sur les paires : paires connectées, compagnons en bout de bras spiral, paires serrées, paires superposées, puis des multiplets et des chaines.

La deuxième partie traite des études statistiques sur les compagnons des galaxies principales, les paires et les groupes.

\section{INTRODUCTION}

We review the observational data on anomalous redshifts ( $z$ or $c z$ ) in groups of galaxies. Theoretical aspects are left out, being reviewed by G. Burbidge, Pecker and Rees, as well as cluster data, treated by de Vaucouleurs. However we shall go down in population to pairs of galaxies, but not to galaxy-quasar associations, treated by Arp.

In the first part we treat individual studies and in the second, statistical ones. In the first we start with pairs of galaxies : connected pairs, companions on ends of spiral arms, close pairs and superimposed pairs, ending with multiplets and chains. 
In the statistical part we deal with the question of companions to main galaxies, then with pairs and groups.

\section{PART I : INDIVIDUAL STUDIES}

\section{CONNECTED PAIRS.}

Pairs of galaxies with different redshifts and connected by luminous filaments have attracted much attention, particularly the $\mathrm{Ma} 2 \mathrm{O} 5$ and NGC 7603 cases.

Ma 205 : with $\mathrm{cz} \sim 21000 \mathrm{~km} \mathrm{~s}^{-1}$, the compact galaxy is 40 " south of the spiral NGC 4319 with $\mathrm{cz}=1800 \mathrm{~km} \mathrm{~s}$. Arp (1971 b, 1973 d), Arp et al. (1975) provided several photographs showing a straight filament connecting the two galaxies, both in continuum light and in H $\alpha$. Lynds and Millikan (1972) also found a connection in continuum but ascribed it to overlapping of the galactic images adding up with an other object (a galaxy ?). But they did not find a connection in $H \propto$. Ford and Epps (1972) did not find it in $\mathrm{H} \propto$ nor in wide band, and Adams and Weymann (1972) did not find it in $\mathrm{H} \propto$

In view of the $\mathrm{H} \propto$ contradictory results, a recheck by Arp (1973 a) of his $\mathrm{H} \propto$ filter showed that it transmitted also at long wavelengths. He then took a photograph in the 7000-9000 $\mathrm{A}$ band, together with a second one (his figures 7 and 8) which faintly show the filament.

This was redone by Walker et al. (1974) in the same band, but they find no trace of connection down to $2-3 \%$ of the night sky. But according to Arp (1975), Walker et al's cathode has an E-W gradient which makes it difficult to judge and, because the night sky is brighter at Lick, their photograph might show less. 
Willis (1976) has a Westerbork $21 \mathrm{~cm}$ continuum map which shows Ma 205 and a few nearby sources but not NGC 4319 nor a bridge.

Thus agreement is not yet reached on this important case.

NGC 7603 : a disturbed Seyfert spiral, NGC 7603, with cz $\sim 900 \overline{\mathrm{km} \mathrm{s}^{-1}}$, is connected to a bright core and faint halo galaxy with $\mathrm{cz} \sim 17000 \mathrm{~km} \mathrm{~s}^{-1}$; the connection is made up of a curved bridge or spiral arm and a fainter, more diffuse, more curved one ; there is a bright rim at the point where the spiral arm enters the halo of the companion. In view of these peculiarities, one must accept the fact that both galaxies are at the same distance (Arp 1971 a). The main arm has a fainter continuation beyond the companion (Arp 1975).

Walker et al. (1974) confirm both arcs; they have some indication that they may extend beyond the companion, but when they correct for a higher cathode sensitivity, there is no such continuation. If they substract the filament and a faint star nearby, they find no indication of the rim in the halo down to $1 \%$ of night sky, thus no evidence, according to them, of interaction between connection and companion.

This important case is not agreed upon either.

Other cases : NGC $772\left(\mathrm{cz}=2400 \mathrm{~km} \mathrm{~s}^{-1}\right)$ has two companions at $\mathrm{cz} \sim 20000 \mathrm{~km} \mathrm{~s}^{-1}$, one with a connecting filament, one near a protuberence of outer isophote (Arp $1970 \mathrm{~b}$ ); but the field is rich in galaxies.

A galaxy $10^{\prime} \mathrm{NW}$ of NGC 4151, with $\mathrm{cz} \sim 36000 \mathrm{~km} \mathrm{~s} \mathrm{~s}^{-1}$, having a filament with 3 compact objects with $c z \sim 58000 \mathrm{~km} \mathrm{~s}^{-1}$ is reported by Arp at this meeting. 
I ZW 96, an E galaxy, has 2 opposite curved filaments with 2 condensations each; one of these is a compact galaxy with $\Delta \mathrm{cz}{ }^{*}=200 \mathrm{~km} \mathrm{~s}^{-1}($ Arp $1972 \mathrm{~b})$.

3C 371, a radio galaxy with stellar nucleus and halo is at the center of a loose chain of compact galaxies; this halo has a long, straight, extension reaching a pair of these compacts, whose brightest has $\Delta \mathrm{cz}=300 \mathrm{~km} \mathrm{~s}^{-1}$ (Arp $1970 \mathrm{c}$ ).

These two last cases are clear cut physical connections; however the $\Delta \mathrm{cz}$, though positive, are small and could just be of kinetic origin. Maybe they should be joined to the cases of the following paragraph.

\section{COMPANIONS ON ENDS OF SPIRAL ARMS.}

Arp (1970a) pointed out the special case provided by companions which are, at least apparently, on the ends of spiral arms, of the M 51 type. He (1969) investigated 5 such systems and gave $\Delta_{c z}=84,90$ and $180 \mathrm{~km} \mathrm{~s}^{-1}$ for 3 of them (Arp 58, 82 and 87) and Bertola and d'Odorico (1973) and Benvenuti et al. (1976) - 298 $\mathrm{km} \mathrm{s}^{-1}$ for a fourth one (Arp 86). The value for $M 51$ is $132 \mathrm{~km} \mathrm{~s}$ (Burbidge and Burbidge 1964). The mean value is $38 \mathrm{~km} \mathrm{~s}^{-1}$, not significantly positive.

A supplementary case has been published by Danziger and Shuster (1974), NGC 646, with $\Delta \mathrm{cz}=-107 \mathrm{~km} \mathrm{~s}^{-1}$.

An interesting case is provided by NGC 4156 which also lies at the end of a spiral arm (of NGC 4151) and has $\Delta \mathrm{cz} \sim 6000$ km $\mathbf{s}^{-1}$ (Arp, this meeting).

* $\Delta \mathrm{cz}$ is $\mathrm{cz}$ of main galaxy minus $\mathrm{cz}$ of companion. 


\section{CLOSE PAIRS}

Unconnected close galaxies candidates for anomalous redshifts are a blue object with $\mathrm{cz}=35000 \mathrm{~km} \mathrm{~s}^{-1}, 5^{\prime}$ from the peculiar galaxy NGC 520, with cz $=2000$ (Arp et al. 1974) and NGC 6438, an SO galaxy with $\mathrm{cz}=6300 \mathrm{~km} \mathrm{~s}^{-1}$ at $7 \mathrm{kpc}$ from a peculiar irregular having $c z=2700$ in one part and 4300 in the other (Sersic 1966) ; this last case may be an exceptional ejection or be due to a collision with an intergalactic gas cloud (Freeman and de Vaucouleurs 1974).

\section{SUPERIMPOSED PAIRS.}

Arp (this meeting) reports on two cases, NGC 1199 and 5296, which, according to him, have each a compact galaxy in front of them with $\Delta \mathrm{cz} \sim 24000$ and $10000 \mathrm{~km} \mathrm{~s}^{-1}$. Such cases are of course of the utmost interest.

\section{MULTIPLETS}

We review now the case of small compact groups of galaxies or multiplets involved in the anomalous $-\mathrm{z}$ question.

Zwicky Triplet : Three spirals are connected by a luminous filament ; two have cz $7000 \mathrm{~km} \mathrm{~s}^{-1}$ while the third, IC 3483, has $\mathrm{cz} \sim 100 \mathrm{~km} \mathrm{~s}^{-\mathrm{I}}$ (Zwicky 1956); the bridge appears as a tangential continuation of one of the arms of IC 3483, which is unresolved and appears as a giant spiral. In case it is close, then probably in the Virgo cluster, it is a most unusual dwarf. This is the most striking candidate for anomalous negative redshift, but it has not been investigated for 20 years. 
Stéphan quartet : discovered as a quartet of galaxies by Stéphan, it is actually a quintet on the sky, one of the galaxies being dauble ; NGC $7317,18 \mathrm{~A}, 18 \mathrm{~B}$ and 19 have $\mathrm{cz} \sim 7000 \mathrm{~km} \mathrm{~s} \mathrm{~s}^{-1}, 7320$ has $\mathrm{cz} \sim 1000 \mathrm{~km} \mathrm{~s}^{-1}$.

Tammann (1970) finds NGC 7320 resolved on Arp's photograph, and to have same cz as nearby large spiral NGC 7331, which, according to Holmberg's statistical study, should have 4 physical companions. He thus puts 7320 at $\sim 10 \mathrm{Mpc}$, which is confirmed by Allen's (1970) determination of mass and mass to luminosity ratio from $21 \mathrm{~cm}$ observations at Nançay, and later evaluated as 15 Mpc by Balkowski et al. (1973) and Shostak (1974 a).

Balkowski et al. (1974) later attribute NGC 7320, 7331, Shostak's Anon and DDO 213 to a same physical group, probably bound, followed in that by Materne and Tammann (1974), while Lynds (1972), by finding more galaxies at $c z \sim 7000 \mathrm{~km} \mathrm{~s}^{-1}$, confirms the existence of the background cluster suspected by Tammann.

Meanwhile Arp (1973 b) finds that the H II regions in 7320 and $7318 \mathrm{~B}$ have the same angular diameter distributions and promotes the idea that all the quintet is at $\sim 15 \mathrm{Mpc}$, with anomalous $\mathrm{cz} \sim 6000$ $\mathrm{km} \mathrm{s} \mathbf{s}^{-1}$ for the quartet part.

To check this, Balkowski et al. (1973) use five distance criteria based on $21 \mathrm{~cm}$ line observations and derive a distance $\left(22+\begin{array}{r}15 \\ -9\end{array}\right) \mathrm{Mpc}$

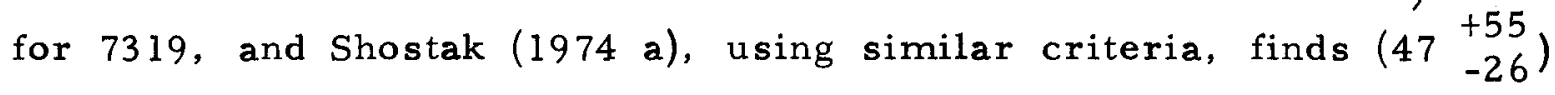
Mpc. The combined result of these two compatible values is in favour of Arp's hypothesis. However recent Westerbork measurements by Allen and Sullivan III ( this conference), show that the neutral hydrogen is displaced out of 7319 optical image. As Balkowski et al. and Shostak's methods are designed for normal galaxies, they can not now be used for the anomalous 7319. The same applies to Shostak's (1974b) lower limit for the distance of $7319 \mathrm{~B}$.

A strong argument for 7319 to be far is its 1971 supernova (Arp 1973 b). 
What there is for the quartet to be close? Arp's argument about H II regions diameters has been criticized by VorontsovVel'yaminov et al. (1974) on the ground that $H$ II regions may reach $3 \mathrm{kpc}$ sizes and is weakened by Sandage and Tammann (1974) who have shown that their size depends on the luminosity of their galaxy. This in turn is criticized by de Vaucouleurs for the case of HII rings. Also, NGC 7319 has only one small visible H II region (Arp 1973 b).

The fact that $H$ II regions are facing each other in $7318 \mathrm{~B}$ and 7320 is no more an argument for interaction, as shown by Hodge (1975), but it is neither an argument against interaction.

The luminous trail S-E of 7320 (Arp 1973 b) is a sign of interaction; one may be tempted to attribute it to 7331 tidal effect, but its distance may be too large. If may also be a prolongation of a feature starting from the quartet and passing behind 7320. Arp intends to obtain its spectrum.

The set of faint filaments found by Arp and Kormendy (1972) deserve attention : are they due to interaction with 7331 , are they galactic foreground ?.

The excess of radio sources found in the region (Arp 1972 a, Gillespie 1974, Kaftan-Kassim and Sulentie 1974) may be attributed to the peculiar nature of the quartet, through still unknown processes; except for 7331 and the quartet, they have no identification with galaxies, but may be with stellar objects which should be thoroughlyinvestigated with large telescopes. The broad, unresolved radio ridge, especially north of the quartet (Kaftan-Kassim and Sulentic 1974) is not confirmed by von Kep-herr et al. (1976).

Other data could fit more easily in internal interactions in the quartet : the arc of continuum radiation found by $\mathrm{Allen}$ and Hartsuiker (1972) may be due to a collision between 7318 and 7319 ; also the straight $H \propto$ filament found by $\operatorname{Arp~(1973~b)~between~} 7319$ and $7318-20$. 
The evidence for anomalous $z$ in Stephan quartet has somewhat weakened, to the profit of a very puzzling multiplet whose further study of nearby radio stellar candidates might reveal other anomalous $z^{\prime} \mathbf{s}$.

Seyfert Sextet : data on this multiplet are given by E. Burbidge and Sargent (1971): 4 galaxies have cz $4400 \mathrm{~km} \mathrm{~s}^{-1}$ while the " $d$ " one has 20 OOO. If $\underline{d}$ is in the group, it is an extremely small size Sc. It is not distorted and Walker et al. (1974) find no residual luminosity between $\underline{d}$ and the other galaxies down to $3 \%$ of sky background and think it to be far behind the quintet.

VV 166 : investigated by Kormendy and Sargent (1974), this octet has 6 galaxies at $6900 \mathrm{~km} \mathrm{~s}^{-1}$ and one, NGC 68, at 5900 . NGC 68 shows no tidal distorsion and its profile follows de Vaucouleurs' $r^{1 / 4}$ law farther than the mean separation of the components; it is also the heavier member. Thus it should not be associated.

\section{CHAINS}

Chains are a particular case of multiplets, forming a more or less wavy linear string of galaxies. A further interest was given to then as, dynamically, they should be short lived configurations. However Turner and Sargent (1973) have shown that a 6-body (stable) group should appear as a loose chain for $8 \%$ of the time.

The best candidate chain for a discrepent $z$ is VV 172 , a string of fixe galaxies, 4 with $\overline{\mathrm{cz}}=16000 \mathrm{~km} \mathrm{~s}^{-1}$, one with $\mathbf{c z}=37$ OOO (Sargent 1968) which is bluer (see bottom of Sandage's Table 3 in Astrophys. J. 183, 711 (1973).

An other one, with 5 galaxies and some more smaller ones nearby, is VV 159, in which two have $\overline{c z} \sim 10000$ and one $13000 \mathrm{~km} \mathrm{~s}^{-1}$ (E. Burbidge and Sargent 1971). 
Two others are given by Arp (1973 a) : the NGC 5128 chain with $\Delta \mathrm{cz}$ up to $3300 \mathrm{~km} \mathrm{~s}^{-1}$ and the NGC $4038-9$ one with $\Delta \mathrm{cz}=$ $14000 \mathrm{~km} \mathrm{~s}^{-1}$.

Bahcall (1973) has shown statistically that objects like the ones reviewed in this first part, even with a connection (but without sign of interaction nor clear other evidence) may be the results of chance superpositions.

As for Stéphan quintet, Arp (1973 c) pointed out that some chains might be physically related to nearby large spirals : VV 150, a chain of 4 with $\mathrm{czN} 8000 \mathrm{~km} \mathrm{~s}^{-1}$ close to NGGC $7318(\mathrm{cz} \sim 1000$ $\mathrm{km} \mathrm{s} \mathrm{s}^{-1}$ ) and a chain of 5 with $\mathrm{cz} N 6000 \mathrm{~km} \mathrm{~s} \mathrm{~s}^{-1}$ close to NGC 247 (cz N 10O). However Balkowski and Chamaraux (1975), using $21 \mathrm{~cm}$ line distance criteria and J.Heidmann's luminosity-diameter relation, have shown that this last chain is at its cosmological distance.

\section{PART II : STATISTICAL STUDIES}

\section{COMPANION GALAXIES}

Arp (1970a) has shown that out of 13 companion galaxies to the 3 major galaxies $M 31, M 81$ and NGC 5128, 11 had z's larger than the ones of the corresponding main galaxy.

A systematic study of this phenomenon made by Bottinelli and Gouguenheim (1973) on de Vaucouleurs'groups, with clearly defined criteria, lead to a mean excess $\overline{\Delta c z}=90 \mathrm{~km} \mathrm{~s}^{-1}$.

When omitting early galaxies, for which a systematic excess of up to $70 \mathrm{~km} \mathrm{~s}^{-1}$ may come from the measurements (de Vaucouleurs and de Vaucouleurs 1972, Simkin 1972), $\overline{\Delta \mathrm{cz}}$ reduces to $60 \mathrm{~km}$ $\mathbf{s}^{-1}$ for 34 companions, with a $5 \%$ probability to be zero or less. Background contamination was estimated negligeable from the lack of clear correlation of excess velocity $\Delta$ cz versus excess magnitude $\Delta \mathrm{m}$ and from galaxy counts. 
However Burke and Hartwick (1974) remarqued that a $\Delta c z-$ $\Delta \mathrm{m}$ correlation could be hidden by spread in absolute magnitude ; instead they found a $\Delta \mathrm{cz}-\Delta \mathrm{r}$ correlation at the $80 \%$ level, estimating the distances $\mathbf{r}$ from van den Bergh luminosity classes. Further, the use of galaxy counts is valid only for real groups, not for "groups" selected a posteriori from accidental projection nor for dissipating groups, nor for wide groups (widening cone effect).

According to them, depth effects (the brightest is biased to be the closest) may have been underestimated by Bottinelli and Gouguenheim, also because of luminosity spread; an intrinsically faint galaxy could nearly apparently match the brightest and disqualify a group, but not the reverse.

Further omitting our Galaxy group and two others, doubtful (?) members and SO galaxies wrongly classified as $S$ by de Vaucouleurs (but without details given), Harrison (1975) is left with 17 non ESO's with a $\bar{z}$ not significantly different from zero.

According to him, transverse velocities of order $200 \mathrm{~km} \mathrm{~s}^{-1}$, for separations larger than $6^{\circ}$, would lead to a $\overline{\Delta \mathrm{cz}}=58 \mathrm{~km} \mathrm{~s}^{-1}$ for Arp's sample, which however deals only with very nearby groups, unlike Bottinelli and Gouguenheim's one.

At this Conference, Arp brought new highly significant data for the $M 31$ and $M 81$ groups. However, viewed the other way round, there is large probability for the (only) two main galaxies to have z lower than the mean $z$ of their companions. Furthermore Bottinelli et al. (1972) have shown that the groups of our Galaxy, M 31, Maffei 1 and M 81 may form a long chain in space and in this respect one should note on Heidmann's (1972) map of this system that, for each group, the main galaxy is on the edge of the corresponding swarm of their companion galaxies. Such a special setting might weaken Arp's argument. Note also that the Maffei 1 companions have a negative $\overline{\Delta c z}$. 


\section{STATISTICS ON PAIRS AND GROUPS}

Anomalous z's have been searched for statistically by Jaakkola (1971) in pairs, groups and clusters. According to our title, we leave out the cluster cases. From Jaakkola's Figure 1, based on Karachentsev's pairs and groups, one gets the following positive and negative (relative) $\Delta \mathrm{Cz}$ for $E, S O$ and $S$ galaxies in pairs and in groups : pairs : $\mathrm{E}$ SO $\mathrm{S}$; groups : $\mathrm{E}$ SO $\mathrm{S}$

$\begin{array}{llllll}+ & 49 & 22 & 21 & 59 \\ - & 568 & 24 & 30 & 50\end{array}$

Thus there is no significant effect with morphological type.

These pairs and groups were later searched by Jaakkola(1976a) for a magnitude-redshift correlation within each system, leading to overall regression coefficients significant to the 1.8 and $0.7-\sigma$ levels respectively.

Jaakkola (1973) also looked, for the same pairs and groups, for relations between excess resdhift $\Delta \mathrm{cz}$ and various other parameters: mean surface brightness, bulge surface brightness, sharpness of galactic edge, light concentration, compactness and inclination. He finds the following effects :

- in pairs, the ones with higher mean surface brightness have higher $z$ 's, at the $1.8-\sigma$ level;

- 17 out of 28 compact galaxies have a positive excess ; in Jaakkola (1976 b) it is 27 out of 38 galaxies ;

- for $\mathrm{Sb}$ and $\mathrm{Sc}^{\prime} \mathrm{s}$ with low bulge surface brightness, 56 out of 77 galaxies have positive excess.

All other relations searched for are not significant.

Jakkola and Molès (1976) investigated de Vaucouleurs'groups. They obtain a 3- $\boldsymbol{\sigma}$ excess velocity for Sbc galaxies, reinforced by a $1-\sigma$ excess for the neighbouring Sc type and, to a smaller extent, by a $0.5-\sigma$ excess for Sb's. For other types, the values are randomly scattered (with the constraint : grand average $=0$ ). 
As there are 11 morphological type bins, the a posteriori probability for chance occurence of this excess is 11 times larger than the a priori one given by Jaakkola. His first study (1971) did not show a significant excess for Sbc-Sc galaxies in groups : 17 negative and 22 positive $\Delta c z$ 's, through, according to him and Molès, de Vaucouleurs' groups are not identical with Karachentsev's ones, with the same elements.

Thus excess redshift for Sbc-Sc's should be looked upon with caution. As pointed out by Joss et al. (1976) : what is the probability to find (on the Sky Atlas)two stars brighter than 9 mag. within $7^{\prime}$ of a galaxy brighter than $8 \mathrm{mag}$ ? Only $810^{-3}$; and they found such a case. But they found it first ; and they calculated the probability after.

\section{REF ERENCES}

Adams, T.F., Weymann, R. J., 1972, Astrophys. Let., 12, 143 Allen, R. J., 1970, Astron. Astrophys. , 7 , 330 Allen, R. J., Hartsuiker, J.W., 1972, Nature, 239, 324

Arp, H., 1969, Astron. Astrophys. , 3, 418

Arp, H., 1970 a, Nature, 225, 1033

Arp, H. , 1970 b, Astrophys. Let. , 5, 257

Arp, H., 1970 c, Astrophys. Let. , 5, 75

Arp, H. , 1971 a, Astrophys. Let. , 7, 221

Arp, H., 1971 b, Astrophys. Let., $\frac{7,}{91}$

Arp, H., 1972 a, Astrophys. J., 174, L 111

Arp, H., 1972 b, External galaxies and quasistellar objects, 380

Arp, H. , 1973 a, Redshift. Controversy., 15

Arp, H. , 1973 b, Astrophys. J. , 183, 411

Arp, H., 1973 c, Astrophys. J., 185, 797

Arp, H., 1973 d, Formation and Dynamics of Galaxies, 199

Arp, H., 1975, Publ. Astron. Soc. Pacif., 87, 545

Arp, H. , Kormendy, J., 1972, Astrophys. J., 178, L 101

Arp, H. , Pratt, N. M. , Sulentic, J. W., 1975, Astrophys. J. , 199. 565

Arp, H. , Sargent, W. L. W. , Khachikian, E. Ye. Andreasion, N. K. , 1974, Astrofizica, 10,298

Bahcall, J., 1973, Redshift. Controversy., 61

Balkowski, C., Bottinelli, L., Chamaraux, P. , Gouguenheim, L., Heidmann, J., 1973, Astron. Astrophys., 25, 319

Balkowski, C., Bottinelli, L., Chamaraux, P., Gouguenheim, L. , Heidmann, J., 1974, Astron. Astrophys., 34, 43 
Balkowski, C., Chamaraux, P., 1975, Astron. Astrophys., 43, 297

Benvenutti, P., d'Odorico, S., Vettolani, P., 1976,

Proc IIIrd Europ. Astron. Meeting. , 393

Bertola, F., d'Odorico, S., 1973, Astrophys. Let., 13, 161

Bottinelli, L., Gouguenheim, L., Heidmann, J., 1972,

Astron. Astrophys. , 18, 121

Bottinelli, L., Gouguenheim, L., 1973, Astron. Astrophys. , 26, 85

Burbidge, E. M. , Burbidge, G. R. , 1964, Astrophys. J., 140, 1445

Burbidge, E. M. , Sargent, W. L. W. , 1971, Nuclei of Galaxies, 351

Burke, J. A., Hartwick, F. D. A., 1974, Astron. Astrophys. , 34, 445

Danziger, I. J., Shuster, H. E., 1974, Astron. Astrophys. 34, 301

Ford, H. C., Epps, H. W. , 1972, Astrophys. Let., 12, $\frac{34}{139}$

Freeman, K.C., de Vaucouleurs, G. , 1974, Astrophys. J., 194, 569

Gillespie, A. R., 1974, Mon. Not. Roy. Astron. Soc. , 166, $11 \mathrm{P}$

Harrisson, E. R., 1975, Astrophys. J., 195, L 61

Heidmann, J., 1972, 1'Astron., $\underline{86,} 257$

Hodge, P. W., 1975, Astrophys. J., 202, 619

Jaakkola, T. , 1971, Nature. , 234, $\overline{534}$

Jaakkola, T., 1973, Astron. Astrophys., 27, 449

Jaakkola, T., 1976a, Proc. III. rd. Europ. Astron. Meeting. , 218

Jaakkola, T., 1976b, Proc. III. rd. Europ. Astron. Meeting., 488

Jaakkola, T., Molès, M. , 1976, Astron. Astrophys., in press

Joss, P.C. , Smith, D. A., Solinger, A. B. , 1976,

Astron. Astrophys. , 47, 461

Kaftan - Kassim, M. A., Sulentic, J. W., 1974,

Astron. Astrophys, , 33, 343

Kep-herr, von A., Haslam, C. G. T. , Wielebinski, R. , 1976, in preparation

Kormendy, J., Sargent, W. L. W. , 1974, Astrophys. J. , 193, 19

Lynds, C.R., 1972, External galaxies and quasistellar objects, 376

Lynds, C. R., Millikan, A. G., 1972, Astrophys. J., 176, L 5

Materne, J., Tammann, G.A., 1974, Astron. Astrophys., 35, 441

Sandage, A., Tammann, G. A., 1974, Astrophys. J. , 190, $\frac{35}{525}$

Sargent, W. L. W., 1968, Astrophys. J., 153, L 135

Sersic, J. L., 1966, Zs.f. Ap. , 64, 202

Shostak, G.S., 1974a, Astrophys. J., 187, 19

Shostak, G.S., 1974b, Astrophys. J., $\underline{189}$, L 1

Simkin, S. M., 1972, Nature., 239, 43

Tammann, G. A., 1970, Astrophys. Let, , 7, 111

Turner, E. L., Sargent, W. L. W. , 1973, Publ. Astron. Soc. Pacif. 85, 538

Vaucouleurs, G. de, Vaucouleurs, A. de, 1972, Nature, 236, 166

Vorontsov-Vel'yaminov, B. A., Efremov, Yu. N. , Komberg, B. V.,

1974, Inst. Prikladnoy Mat. Akad. Nauk., preprint 4

Walker, M. F., Pike, C. D., Mc Gee, J. D. , 1974 ,

Astrophys, J. 194, L 125

Willis, A. G., 1976, Private communication

Zwicky, F., 1956, Erg. Exact. Naturwiss. 29, 344 


\section{DISCUSSION}

T.S. JAAKKOLA: In the study of the nature of redshifts one must not neglect data which is at $2 \sigma$ or even $1 \sigma$ level. Although not proving anything such data gives direction for further checks. We have a difficult problem. If these effects were always at $5 \sigma$ or $9 \sigma$ levels, there would not be any redshift problem; it had already been solved. And if one extracts arbitrarily some particular data out of their context and makes superficial inferences, one will not learn anything about the observations one is looking at. For instance, it is well-known for 20 years that $\mathrm{Sa}$ galaxies, in the Virgo cluster, do not show any redshift in excess of that of $\mathrm{E}$ and $\mathrm{SO}$ galaxies. This is quite natural since the nuclear bulges of $\mathrm{Sa}$ spirals are in several respects mach like ellipticals. A low statistical significance of an effect in a single system or in one category of systems does not prove anything about the value of some set of evidence. Chance probability of an effect occurring at a lower significance but in the same direction in clusters, groups and pairs can be multiplied and one can in fact have probabilities, say $10^{-10}$. My results on the magnitude and surface brightness relations of redshift (Astron. Astrophys. 27, 449, 1973; 449, 1973; Proc. III European Astron. Meeting, p. 218, Tbilizi 1976) are significant at $99 \%$ level, not using that combination method.

Concerning in particular the typeredshift relation, recent results by me and Moles are shown in the figure. This study confirms, at a $3 \sigma$ level of significance, in de Vaucouleurs' groups of galaxies the earlier result (Jaakkola, Nature 234, 534, 1971) found for clusters and Karachentsev's groups. $\mathrm{Sb}$ and $\mathrm{Sc}$ galaxies have larger redshifts than galaxies of the other types, with the mean difference $130 \pm 43 \mathrm{~km} / \mathrm{s}$. The possibility that the effects were due to

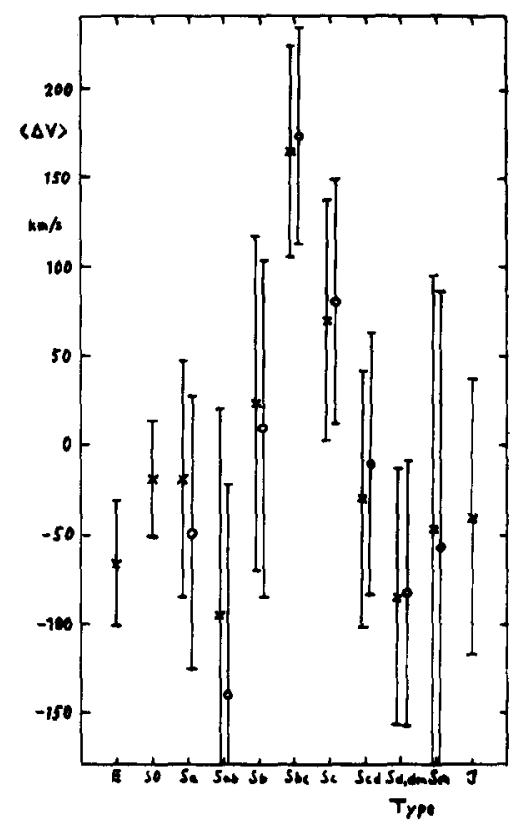


observational errors or selection effects is extensively discussed in our report (Astron. Astrophys., in press), and the explanation can probably not be found along these lines.

J. HEIDMANN: As I said, my review does not include clusters, and for groups, your 1971 study does not show a significant excess.

W.T. SULLIVAN: It seems reasonable to Dr Allen and myself that NGC 7320 is a dwarf galaxy, not unlike M 33 in its properties, whereas NGC 7318 B is an Sc I, not unlike M 101, but at a distance about 7 times further away. The linear sizes of the largest $\mathrm{H}$ II regions are then $\sim 300 \mathrm{pc}$ and $2-3 \mathrm{kpc}$ respectively, similar to $\mathrm{M} 33$ and $\mathrm{M} \mathrm{101.} \mathrm{The} \mathrm{total} \mathrm{mass} \mathrm{of}$ HI measured in the two redshift ranges is also very similar to that measured in M 33 and M 101 if the HI is placed at its "Hubble distance". The presence of other galaxies at both redshifts in the larger field of several degrees in size adds to the probability that two superimposed groups of galaxies happen to give a very close coincidence in Stephan's Quintet.

I would also like to take this opportunity to report on some observations which I conducted several years ago with the Westerbork telescope. The object was to search for any radio peculiarities, especially bridges or extended emission, which might be associated with several pairs of apparently nearby objects exhibiting discrepant redshifts. The following pairs were examined (pair no. 5 being of interest because the QSS and nearby cluster of galaxies have identical redshifts):

\begin{tabular}{|c|c|c|c|c|c|}
\hline \multirow{2}{*}{ No. } & Objects & Types & $\underline{\text { Redshifts }}$ & \multicolumn{2}{|c|}{$\begin{array}{l}\text { Approx } \\
\text { Separation Comments }\end{array}$} \\
\hline & 3C $275.1 / N 4651$ & QSS/Sc & $0.56 / 0.0025$ & $4^{\prime}$ & $\begin{array}{l}\text { Photo in Sandage } \\
\text { et al } 1965, \text { Astro- } \\
\text { phys. J., } 142 \text {, } \\
\text { 1307. }\end{array}$ \\
\hline 2. & $3 \mathrm{C} 309.1 / \mathrm{N} 5832$ & $\mathrm{QSS} / \mathrm{SBb}$ & $0.90 / 0.0020$ & $6^{\prime}$ & \\
\hline 3. & Mark 205/N4319 & $\begin{array}{l}\text { compact } \\
\text { object/s }\end{array}$ & $0.070 / 0.005$ & $I^{\prime}$ & $\begin{array}{l}\text { Disputed optical } \\
\text { bridge; see Arp } \\
\text { 1973, IAU Symp. } 58\end{array}$ \\
\hline 4. & $1108+285 / N 3561$ & QSO/pec.gal & $2.19 / ?$ & $2^{\prime}$ & $\begin{array}{l}\text { Photo in Arp 1971, } \\
\text { Science, } 174,1189 .\end{array}$ \\
\hline 5. & S225I+11/Anon & $\begin{array}{l}\text { QSS/Cluster of } \\
\text { galaxies }\end{array}$ & $0.32 / 0.32$ & $I^{\prime}$ & $\begin{array}{l}\text { See Gunn 1971, } \\
\text { Astrophys. J.' } \\
164, \mathrm{LI73}\end{array}$ \\
\hline
\end{tabular}


In all cases the available angular resolution was more than adequate to separate the two objects, but the effective sensitivity to weak emission was somewhat reduced for the three cases in which a strong QSS was in the field (dynamic range problems). The results for the five respective cases were as follows:

(1) observed at $\lambda 21-\mathrm{cm}$; no extended emission above $\sim 20 \mathrm{mJy}$ (including galaxy disk); QSS $=2.9 \mathrm{Jy}$.

(2) observed at $\lambda 21-\mathrm{cm}$; no extended emission above $\sim 200 \mathrm{mJy}$; QSS $=8.6 \mathrm{Jy}$.

(3) observed at $\lambda 21-\mathrm{cm}$ (data made available by P.C. van der Kruit; see Astron. \& Astrophys., 15, 110, 1971); van der Kruit's finding of no radio emission ( $<3 \mathrm{mJy}$ ) associated with either of the listed objects confirmed; Arp's corrected optical ID of a $12 \mathrm{mJy}$ radio source (RS) $\sim 1: 5 \mathrm{~N}$ of $\mathrm{N} 4319$ also confirmed; 6 RSs found within $\sim 40^{\prime}$ of $\mathrm{N4319}$, of which only one (at a distance of 18, $\mathrm{N}$ ) had an optical ID. This was an $80 \mathrm{mJy}$ source coinciding in position with a very faint galaxy and located at $\alpha(1950)=12^{\mathrm{h}} 21^{\mathrm{m}} 21^{\mathrm{s}}, \delta=+75^{\circ} 53 ! 1, \sim 6^{\prime}$ NW of the $13^{\mathrm{m}} \mathrm{E}$ galaxy $\mathrm{N} 4386$.

(4) observed at $\lambda 21-\mathrm{cm}$ (data made available by G.K. Miley); QSO shows no emission > $3 \mathrm{mJy}$; peculiar galaxy N3561, however, exhibits two concentrations of radio emission coincident in form and position with the double optical structure; northern concentration has flux density of $10 \mathrm{mJy}$ and southern $\sim 30 \mathrm{mJy}$; no identified RSs in the field despite the high density of nearby galaxies.

(5) observed at $\lambda 6 \mathrm{~cm}$ (HPBW 6" $\times$ 40" ( $\alpha \times \delta)$ ); no radio emission in vicinity of the small cluster of galaxies above $10 \mathrm{mJy}$ level; QSS $=0.6 \mathrm{Jy}$. In summary, all pairs gave negative results in terms of any bridges or extended radio emission, although the radio structure of $\mathrm{N} 356 \mathrm{I}$ is very interesting in itself.

J.I. SERSIC: Some years ago, Mrs Burbidge has demonstrated the nonexistence of the $6000 \mathrm{~km} / \mathrm{s}$ component in NGC 6438. Further plates taken at Cordoba. confirm this fact.

H. ARP: Stephan's Quintet will be discussed further tomorrow but I want to make one remark about HI distance of chains. If the chains are closeby then they are intrinsically small, if they are intrinsically small they 
presumably contains small amounts of hydrogen. In that case applying normal HI distance criteria would naturally give large distances.

Actually the distribution of these chains is the most significant fact. I would challenge the observers to product chains like Stephan's Quintet, VVI50 and the chain near NGC 247, which are not near large, low redshift galaxies.

B.M. LEWIS: In addition to the studies you have mentioned of discrepant redshifts in groups, there is my 1971 study of de Vaucouleurs' groups, and of Page's double galaxies, which gave null results.

The existence of discrepant redshifts as a result of centrally dependent effects, is tested by comparing $21 \mathrm{~cm}$ and optical estimates of the systematic velocities of galaxies. These limit any effect to less than about $10 \mathrm{~km} \mathrm{~s}^{-1}$.

L. GOUGUENHEIM: What we did was somewhat different because we restricted our study to the de Vaucouleurs' groups and, within these groups, to the galaxies having "accurate" velocity determinations (as given in the Reference Catalogue of Bright Galaxies). However, further studies of these radial velocities, such as Lewis published in the Mon.Not.Roy.Astr.Soc. or that we published in Astron. Astrophys. show that in several cases the errors quoted on the optical radial velocities are too optimistic.

P.C. VAN DER KRUIT: 1. Dr. Sullivan's $2 \mathrm{kpc}$ for the HII-region in NGC 7318 seems to me certainly not that easily comparable to the complexes in M 101 , which are at least a factor $2-3$ smaller. Also Arp originally did quote a largest value more around $3 \mathrm{kpc}$.

2. The examples shown by Dr. Heidmann are all drawn from earlier work of Arp's. Quite a few will qualify as ejecting galaxies as he referred to this morning. I would like to ask Dr. Arp whether he also searched for quasars around these systems as he did for the examples he showed this morning.

H. ARP: No.

J.P. VIGIER: As to the statistical discussion of possible redshift - type correlation we have now a possible tool to settle the question, i.e. de Vaucouleurs' new catalogue where optical and $21 \mathrm{~cm}$ velocities are no longer discrepant. Once the groups have been selected therein one will 
be able to remake the work of Jaakkola and Molès (with smaller o's) and see whether we justify their result or not.

G. DE VAUCOULEURS: 1) We have taken sky-limited image tube spectrograms of N 4319 and Markarian 205 with the slit along the suspected bridge, but failed to record any trace of $\mathrm{H \alpha}$ emissions at velocities intermediate between the 2 systems. 2) I should like to remind you that in selecting galaxies as possible group members, any with large redshift discrepancies would have been rejected a priori. 